\title{
BMJ Open Patient-reported outcome measures (PROMs) following knee arthroplasty: a prospective cohort study protocol
}

\author{
Nicole Vogel (D) , ${ }^{1,2}$ Thomas Rychen, ${ }^{2}$ Raphael Kaelin, ${ }^{2}$ Markus P Arnold ${ }^{1,2}$
}

To cite: Vogel N, Rychen T, Kaelin $\mathrm{R}$, et al. Patientreported outcome measures (PROMs) following knee arthroplasty: a prospective cohort study protocol. BMJ Open 2020;10:e040811. doi:10.1136/ bmjopen-2020-040811

- Prepublication history for this paper is available online. To view these files, please visit the journal online (http://dx.doi org/10.1136/bmjopen-2020040811).

Received 22 May 2020 Revised 20 0ctober 2020 Accepted 26 October 2020

Check for updates

(C) Author(s) (or their employer(s)) 2020. Re-use permitted under CC BY-NC. No commercial re-use. See rights and permissions. Published by BMJ.

${ }^{1}$ Faculty of Medicine, University of Basel, Basel, Switzerland

${ }^{2}$ Practice Leonardo, Hirslanden Klinik Birshof, Münchenstein, Switzerland

Correspondence to

Nicole Vogel;

nicole.vogel@unibas.ch

\section{ABSTRACT}

Introduction To evaluate the quality of clinical practice, patient-reported outcome measures (PROMs) are important as certain questions could only be answered by the patient himself. PROMs help to get a better understanding what is meaningful to a patient and directly affects daily functioning. To move beyond traditional measures, we are interested in what matters to patients and developed this project. The aim of this article is to provide the protocol for our study collecting PROMs in daily medical practice from patients who undergo knee arthroplasty.

Methods and analysis This study is a single-site, observational, prospective cohort study. We will recruit patients scheduled for a knee arthroplasty in our medical office, situated in a private clinic. After signed informed consent, patients complete self-reported questionnaires before the surgery, after 4 months, 1 year, 2 years, 3 years, 4 years and 5 years. We will use the following PROMs: Knee injury and Osteoarthritis Outcome Score, Forgotten Joint Score, EuroQol five dimensions and satisfaction. Additionally, the surgeon will complete the objective Knee Society Score. Administration of the questionnaires will be electronically or paper-based. We will assess differences between preoperative and postoperative data with paired t-test for continuous variables and Wilcoxon signedrank test for categorical variables. To assess subgroup differences, we will use unpaired t-test for continuous variables and Mann-Whitney $U$ test for categorical variables. To assess possible presence of bias, we will conduct sensitivity analyses.

Ethics and dissemination The study has been reviewed and approved by the local ethics committee in Basel, Switzerland. Written informed consent will be obtained from all patients. We will disseminate the results of the study through peer-reviewed journals, national and international conference presentations and presentations to relevant stakeholders through appropriate channels.

\section{INTRODUCTION}

Patient-reported outcomes are an important element to evaluate the quality and the results of clinical practice as they directly and without any interpretation report a patient's health status. ${ }^{1}$ Certain questions, for example, regarding health-related quality of life (QoL) or functioning in daily life can only be answered by the patient himself.

\section{Strengths and limitations of this study}

- Our results return a direct feedback from patients with knee arthroplasties, short to long term.

- The findings will help to understand why some patients with knee arthroplasties are not satisfied and can be compared with other data from registries with patient-reported outcome measures (PROMs).

- The Swiss National Joint Registry does not apply PROMs, our study helps to collect important data.

- Participants are recruited from a single private medical office consisting of a team of three subspecialised knee surgeons. The sample size may not be representative for all patients in Switzerland, but is likewise a homogeneous sample.

Patient-reported outcome measures (PROMs) are standardised measures to capture and translate the patients' perspective into objective numerical data. The Organisation for Economic Co-operation and Development (OECD) promotes people-centredness of primary care services ${ }^{2}$ and the International Consortium for Health Outcomes Measurement is developing more standard sets to measure PROMs. ${ }^{3}$

PROMs help to get a better understanding of outcomes that are most meaningful to patients and have direct relevance to their everyday functioning. Patients with arthroplasties differ in what they consider important: the ability to run, kneel or squat has not the same relevance for all patients. ${ }^{4}$ Despite high implant survival rates and good overall outcome of knee arthroplasties, up to $20 \%$ of the patients are still unsatisfied. ${ }^{5}$

Each year, 21000 patients undergo total knee arthroplasty (TKA) in Switzerland. ${ }^{6}$ The frequency of 250 per 100000 population is the highest among the OECD countries. ${ }^{6}$ The Swiss National Joint Registry captures implant specific outcomes of almost all TKA procedures in Switzerland, but until now the registry does not capture PROMs. ${ }^{7}$ To move beyond traditional measures, we are 


Table 1 Inclusion and exclusion criteria
Inclusion
Patients scheduled for elective partial or total
knee arthroplasty.
Age $>18$ years.
Exclusion
$\quad$ Insufficient knowledge of German, English,
Italian or French to understand the consent
form and the questionnaires.

interested in what matters to patients and developed this project. The aim of this article is to provide the protocol for our study collecting PROMs in daily medical practice from patients undergoing knee arthroplasty.

\section{METHODS AND ANALYSIS}

\section{Study design, setting and recruitment}

This study is a single-site, observational, prospective cohort study with a follow-up of five years. Recruitment will take place in our medical office, situated in a private clinic. Three experienced knee surgeons (TR, RK, MPA) will consecutively recruit patients scheduled for elective partial or TKA. As part of daily routine, we will ask all patients eligible to participate. The participation is voluntary and will not affect patients' further treatment. The inclusion and exclusion criteria are described in table 1.

A trained research associate (NV) will be responsible to instruct patients about the needs and goals of the project, to distribute and collect the questionnaires and to manage and analyse collected data.

\section{Procedures and follow-up}

After signed informed consent, we will ask patients to complete the self-reported questionnaires not before 3 weeks before the surgery. Postoperative, we will distribute questionnaires after 4 months, 1 year, 2 years, 3 years, 4 years and 5 years. Questionnaires are predominantly electronically administered or otherwise paperbased, whereby a trained research associate will enter the data into our database. In general, all study data will be collected and managed using Research Electronic Data Capture (REDCap), a secure web-based software hosted on a secured server placed in Switzerland. Authorised study personnel only will have access to uncoded patient data.

During the routine medical screening, surgeons will as well complete a questionnaire: before the surgery, after 4 months, 1 year and 5 years. We will collect demographic data and data concerning surgery details from patients' medical records. Independent of our study, every patient scheduled for a total knee or hip arthroplasty at our clinic, is eligible to participate in the Swiss National Joint Registry. For those patients who agreed to take part in the registry, we will have standardised data on their general state of health, previous surgeries and implant details.

Our data collection will be joint-based, rather than patient-based. Thus, patients with bilateral surgery will complete questionnaires for each knee joint. In case of an unreturned questionnaire, we will send out a reminder or call the patient. To assess external validity, we will collect anonymised information about age and sex for those patients not willing to take part in the study. A detailed summary about outcome measures and their distribution over time is outlined in table 2.

\section{Outcome measures}

For patients with knee osteoarthritis, numerous validated questionnaires are available to assess several aspects of functioning and health-related QoL. Such questionnaires typically comprise items on joint function, pain, stiffness and treatment satisfaction. We screened the literature for international guidelines, ${ }^{38}$ common PROMs in other relevant studies and national arthroplasty registries ${ }^{9}$ and followed the recommendation to combine generic and specific PROMs. ${ }^{10}$ We tried to find a reasonable balance between the amount of PROMs applied and the burden for the patient to answer all items in order to increase our participation rate. As a result, we decided to use three validated PROMs and one validated clinician-completed questionnaire.

\section{Objective Knee Society Score}

The Knee Society Score (KSS) consists of different parts, we will only use the objective KSS. ${ }^{11}$ Four items regarding alignment, stability and joint motion completed by the surgeon and three patient reported items on symptoms contribute to the KSS. The total score ranges from 0 to 100 points with a higher score indicating better outcome. The KSS is valid, responsive, reliable and consistent in patients with arthroplasties. ${ }^{12} 13$

\section{Knee injury and 0steoarthritis Outcome Score}

The Knee injury and Osteoarthritis Outcome Score (KOOS) is developed to assess the patient's opinion about their knee and associated problems. ${ }^{14}$ The KOOS consists of 42 items divided into five subscales on pain, symptoms, activities of daily living, sports and recreational activities and QoL. Each subscale ranges from 0 to 100 points, with a higher score indicating better outcome. A total score has not been validated and is not recommended. Adequate measurement properties have been demonstrated. ${ }^{15}$

\section{Forgotten Joint Score}

The Forgotten Joint Score (FJS-12) measures through 12 items the new concept of patients' ability to forget the artificial joint in everyday life. ${ }^{16}$ The total score ranges from 0 to 100 points with a higher score indication better outcome. The measurement properties have been evaluated and confirmed for patients with arthroplasties in several studies. ${ }^{17-19}$ The FJS-12 showed less ceiling effects than the KOOS and might be more discriminating in patients with TKA. ${ }^{20}$

\section{EuroQol five dimensions three levels}

The three-level version of the EuroQol five dimensions (EQ-5D-3L) is one of the most widely used instruments 
Table 2 Outcome measures and time of completion

\begin{tabular}{|c|c|c|c|c|c|c|c|c|}
\hline \multirow[b]{2}{*}{ Outcome measures } & \multirow[b]{2}{*}{ Instrument } & \multicolumn{7}{|c|}{ Time of completion } \\
\hline & & Before surgery & $\begin{array}{l}4 \\
\text { months }\end{array}$ & $\begin{array}{l}1 \\
\text { year }\end{array}$ & $\begin{array}{l}2 \\
\text { years }\end{array}$ & $\begin{array}{l}3 \\
\text { years }\end{array}$ & $\begin{array}{l}4 \\
\text { years }\end{array}$ & $\begin{array}{l}5 \\
\text { years }\end{array}$ \\
\hline \multicolumn{9}{|l|}{ Patient-reported, knee specific } \\
\hline Symptoms & KOOS symptoms & $x$ & $x$ & $x$ & $\mathrm{x}$ & $x$ & $x$ & $x$ \\
\hline Sports and recreational activities & KOOS sports & $x$ & $x$ & $x$ & $x$ & $x$ & $x$ & $x$ \\
\hline Knee related quality of life & KOOS QoL & $x$ & $x$ & $x$ & $x$ & $x$ & $x$ & $x$ \\
\hline Forgotten Joint & FJS-12 & $x$ & $x$ & $x$ & $x$ & $x$ & $x$ & $x$ \\
\hline Satisfaction with surgery & Likert scale & & $x$ & $x$ & $x$ & $x$ & $x$ & $x$ \\
\hline Health-related quality of life & EQ-5D-3L & $x$ & $x$ & $x$ & $x$ & $x$ & $x$ & $x$ \\
\hline \multicolumn{9}{|l|}{ Clinician-completed } \\
\hline Knee function & KSS & $x$ & $x$ & $x$ & & & & $x$ \\
\hline Degree of osteoarthritis & Kellgren-Lawrence Scale & $x$ & & & & & & \\
\hline Satisfaction with surgery & Likert scale & & $x$ & $x$ & & & & $x$ \\
\hline
\end{tabular}

ADL, activities of daily living; EQ-5D-3L, EuroQol five dimensions three levels; FJS-12, Forgotten Knee Joint Score; KOOS, Knee injury and Osteoarthritis Outcome Score; KSS, Knee Society Score; QoL, quality of life.

for measuring generic health-related $\mathrm{QoL}^{21}{ }^{22}$ and is also the most commonly used generic PROM among arthroplasty registries. ${ }^{23}$ The EQ-5D-3L captures five dimensions (mobility, self-care, usual activities, pain/discomfort and anxiety/depression) based on three levels of severity (no problems, moderate problems, extreme problems). To derive the summary index score, a value set of the general population of a country or region is required. In the absence of a value set for Switzerland, we will apply the European value set. The index score ranges from 0 to 1 with a higher value indication better health.

Additionally, a Visual Analogue Scale (EQ-VAS) records the patient's self-rated health on a vertical VAS with the endpoints 'The best health you can imagine' (100 points) and 'The worst health you can imagine' ( 0 points). The EuroQol five dimensions instruments have been proven to be valid, reliable and responsive in numerous conditions and populations ${ }^{22}$

\section{Satisfaction}

Besides the usage of validated questionnaires, it is recommended to use an extra item to measure global satisfaction. Such an item presents with a good face validity and gives the patient the opportunity to summarise his point of view. ${ }^{10}$ We will ask patients on a five-point Likert scale how satisfied they are with the result of the surgery (very satisfied, satisfied, neutral, unsatisfied or very unsatisfied). To objectify this outcome, surgeons will answer this question as well.
We will also ask patients, if they would undergo the surgery again (yes or no) and how they rate their current overall improvement in their knee joint compared with the state before the surgery on a seven-point Likert scale (very much better, substantially better, a little better, no change, a little worse, substantially worse or very much worse). Health transition items are another type of PROMs to reflect a self-perceived change over a defined period.

\section{Outcomes}

We will calculate the total score for each outcome measure according their published algorithms. For all outcome measures, we will report end and change scores. Our primary outcome will be patients' satisfaction with the result of the surgery; whereas all other patient-reported and clinician-reported outcomes, adverse events and revision surgery will be secondary outcomes.

\section{Sample size and study duration}

The volume of knee arthroplasties at our medical office is approximately 130 arthroplasties in 120 patients per year (bilateral surgery possible). We estimate that only few patients are not eligible because of language barriers as the questionnaires are available in four languages (German, English, Italian and French). We will ask all patients eligible to participate and assume that $20 \%$ will reject. Finally, we will recruit about 100 patients per year and aim for at least a $90 \%$ follow-up rate in the first 2 years 
after surgery. A small proportion (3\%) will be patients with revision surgery whose data will be analysed separately from patients with primary knee arthroplasty.

The recruitment period will be five years, data collection period ten years (fiveyears per patient), respectively. In total, we aim to collect data from 500 knee arthroplasties.

\section{Missing data}

We will regard the instructions on how to handle missing answers for each outcome measure. For each of the KOOS subscales at least half of the answers are mandatory, for the FJS-12 two-thirds are mandatory to calculate a mean score. All answers are mandatory for the EQ-5D-3L to compute the index value. We will report the frequency of missing data for each outcome measure and each follow-up.

\section{Statistics}

We will perform statistical analyses with IBM SPSS Statistics for Windows, Version 26.0. Armonk, NY: IBM Corp. Descriptive statistics will be presented, including means and standard deviation (SD) for all continuous variables, and frequency counts and percentages for categorical variables. As bilateral knee arthroplasties are possible, we will report joint-based data, rather than patient-based data.

The Kolmogorov-Smirnov test will be used to verify normal distribution. To assess differences between preoperative and postoperative data we will use paired t-test for continuous variables and Wilcoxon signed-rank test for categorical variables. To assess subgroup differences, we will use unpaired t-test for continuous variables and Mann-Whitney $\mathrm{U}$ test for categorical variables. To assess possible presence of bias we will conduct sensitivity analyses. We will perform all tests two tailed and consider $\mathrm{p} \leq 0.05$ as statistically significant.

\section{Burden and practical considerations}

We estimated that patients need maximum 10 to $15 \mathrm{~min}$ and surgeons 2 to $3 \mathrm{~min}$ to complete the questionnaires. Questionnaires for patients are available in four different languages and administered electronically or paperbased. For a private medical office, the administrative burden and costs involved in routine collection of PROMs for knee arthroplasty patients are significant. A research associate coordinates all procedures and put the collection of PROMs into daily medical practice. Technical issues needed to be resolved to implement a database that is able to capture study data and send out questionnaires to patients and surgeons.

\section{Ethics and dissemination}

Our study is in accordance with the World Medical Association Declaration of Helsinki ${ }^{24}$ and was approved by the local ethics committee in Basel, Switzerland (reference: 2016-01777).$^{25}$ All patients will have to sign a written informed consent. The findings of the study will be published in peer-reviewed journals and presented at national and international conferences. Moreover, we will communicate the results to relevant stakeholders like patients, clinicians, healthcare providers or policymakers through appropriate channels.

\section{Patient and public involvement}

Patients and the public were not directly involved in planning, design and development of the study. However, the results will be published and publicly available.

\section{DISCUSSION}

We hereby described the methods of our study to collect PROMs from patients undergoing knee arthroplasty. Our aim is to contribute to the implementation of PROMs collection into daily medical routine. For a private medical office, it is a burden regarding costs and time to realise such a project. However, we are interested in the real results of our patients and want to identify, and if possible to reduce, factors leading to unhappy patients after a knee arthroplasty. In addition, we want to answer the question, if different types of implants, off-the-shelf or customised individually made implants, show any differences in their outcomes.

We selected a balanced combination of validated generic und disease specific PROMs that are commonly used and likewise present in national arthroplasty registries. Beyond traditional PROMs like KOOS and EQ-5D-3L, we added the FJS-12. It comprises a relatively new and convincing concept of joint awareness and measures the ability to forget the artificial joint, while having less ceiling effects. ${ }^{26}$ Distribution of questionnaires will predominantly be electronically. Thus, we avoid transcription errors while distribution is more efficient and less time consuming. We are optimistic, that the need for paper administration will diminish with time since also elderly get familiar with new technologies. ${ }^{10}$

Besides the evaluation of the patients perspective, PROMs can be used to engage patients in medical decision making. ${ }^{27}$ Patients may have little experience participating in medical or health decisions and may not recognise the important role they play in clarifying their values and incorporating them into decisions. ${ }^{28}$ In summary, we believe that the results of our study will be useful for all stakeholders, like clinicians, patients or policy-makers to enhance the understanding of the impact of knee osteoarthritis or knee arthroplasty on the patient.

\section{Current study status}

Study recruitment and data collection began in January 2017. In total, recruitment will last for fiveyears and data collection for ten years (last patient last visit). As of the time of submission of the manuscript, we already recruited 310 patients. A continuation of the recruitment and study duration is conceivable and will be applied at the local ethics committee in due time.

Contributors NV, TR, RK and MPA designed the project. NV drafted the manuscript. TR, RK and MPA reviewed and edited the manuscript. All authors approved the final manuscript. 
Funding The authors have not declared a specific grant for this research from any funding agency in the public, commercial or not-for-profit sectors.

Competing interests None declared.

Patient and public involvement Patients and/or the public were involved in the design, or conduct, or reporting, or dissemination plans of this research. Refer to the Methods section for further details.

Patient consent for publication Not required.

Provenance and peer review Not commissioned; externally peer reviewed.

Open access This is an open access article distributed in accordance with the Creative Commons Attribution Non Commercial (CC BY-NC 4.0) license, which permits others to distribute, remix, adapt, build upon this work non-commercially, and license their derivative works on different terms, provided the original work is properly cited, appropriate credit is given, any changes made indicated, and the use is non-commercial. See: http://creativecommons.org/licenses/by-nc/4.0/.

ORCID iD

Nicole Vogel http://orcid.org/0000-0002-8322-4514

\section{REFERENCES}

1 U.S. Department of Health and Human Services Food and Drug Administration. Patient-Reported outcome measures: use in medical product development to support labeling claims. U.S. food and drug administration, 2009. Available: http://www.fda.gov/regulatoryinformation/search-fda-guidance-documents/patient-reportedoutcome-measures-use-medical-product-development-supportlabeling-claims [Accessed 3 Dec 2019].

2 Patient-reported Indicator Surveys (PaRIS) - OECD. Available: http:// www.oecd.org/health/paris/ [Accessed 10 Sep 2020].

3 ICHOM | Healthcare Improvement | Patient-Reported Outcomes. ICHOM - International Consortium for health outcomes measurement. Available: https://www.ichom.org/ [Accessed $10 \mathrm{Sep}$ 2020].

4 Wiering B, de Boer D, Delnoij D. Asking what matters: the relevance and use of patient-reported outcome measures that were developed without patient involvement. Health Expect 2017;20:1330-41.

5 Gunaratne R, Pratt DN, Banda J, et al. Patient Dissatisfaction following total knee arthroplasty: a systematic review of the literature. $J$ Arthroplasty 2017;32:3854-60.

6 OECD statistics. Available: https://stats.oecd.org/Index.aspx? ThemeTreeld=9 [Accessed 3 Dec 2019].

7 Schweizer Implantate-Register SIRIS. SIRIS IMPLAT. Available: http://www.siris-implant.ch/en/Fachinformation/SchweizerImplantate-Register-SIRIS [Accessed 15 Oct 2019].

8 Canadian Institute for Health Information, Organisation for Economic Co-operation and Development. OECD Patient-Reported Indicator Surveys (PaRIS) Initiative: Patient-Reported Outcome Measures (PROMs) for Hip and Knee Replacement Surgery - International Data Collection Guidelines. Ottawa, ON: CIHI, 2019.

9 Wilson I, Bohm E, Lübbeke A, et al. Orthopaedic registries with patient-reported outcome measures. EFORT Open Rev 2019;4:357-67.

10 Rolfson O, Bohm E, Franklin P, et al. Patient-Reported outcome measures in arthroplasty registries report of the patient-reported outcome measures Working group of the International Society of arthroplasty registries Part II. recommendations for selection, administration, and analysis. Acta Orthop 2016;87 Suppl 1:9-23.

11 Noble PC, Scuderi GR, Brekke AC, et al. Development of a new knee Society scoring system. Clin Orthop Relat Res 2012;470:20-32.

12 Culliton SE, Bryant DM, MacDonald SJ, et al. Validity and internal consistency of the new knee Society knee scoring system. Clin Orthop Relat Res 2018;476:77-84.

13 Kayaalp ME, Keller T, Fitz W, et al. Translation and validation of the German new knee Society scoring system. Clin Orthop Relat Res 2019;477:383-93.

14 Roos EM, Lohmander LS. The knee injury and osteoarthritis outcome score (KOOS): from joint injury to osteoarthritis. Health Qual Life Outcomes 2003;1:64.

15 Collins NJ, Prinsen CAC, Christensen R, et al. Knee injury and osteoarthritis outcome score (KOOS): systematic review and meta-analysis of measurement properties. Osteoarthritis Cartilage 2016;24:1317-29.

16 Behrend H, Giesinger K, Giesinger JM, et al. The "forgotten joint" as the ultimate goal in joint arthroplasty: validation of a new patientreported outcome measure. J Arthroplasty 2012;27:430-6.

17 Thomsen MG, Latifi R, Kallemose T, et al. Good validity and reliability of the forgotten joint score in evaluating the outcome of total knee arthroplasty. Acta Orthop 2016;87:280-5.

18 Giesinger K, Hamilton DF, Jost B, et al. Comparative responsiveness of outcome measures for total knee arthroplasty. Osteoarthritis Cartilage 2014;22:184-9.

19 Baumann F, Ernstberger T, Loibl M, et al. Validation of the German forgotten joint score (G-FJS) according to the COSMIN checklist: does a reduction in joint awareness indicate clinical improvement after arthroplasty of the knee? Arch Orthop Trauma Surg 2016;136:257-64.

20 Thompson SM, Salmon LJ, Webb JM, et al. Construct validity and test Re-Test reliability of the forgotten joint score. J Arthroplasty 2015;30:1902-5.

21 EuroQol Group. EuroQol--a new facility for the measurement of health-related quality of life. Health Policy 1990;16:199-208.

22 EuroQol. Available: https://euroqol.org/ [Accessed 24 Oct 2019].

23 Rolfson O, Eresian Chenok K, Bohm E, et al. Patient-Reported outcome measures in arthroplasty registries. Report of the patientreported outcome measures Working group of the International Society of arthroplasty registries Part I. overview and rationale for patient-reported outcome measures. Acta Orthop 2016;87:3-8.

24 WMA - The World Medical Association-WMA Declaration of Helsinki - Ethical Principles for Medical Research Involving Human Subjects. Available: https://www.wma.net/policies-post/wma-declaration-ofhelsinki-ethical-principles-for-medical-research-involving-humansubjects/ [Accessed 28 Nov 2019].

25 Swissethics. Registry of all projects in Switzerland (RAPS). Available: https://ongoingprojects.swissethics.ch/runningProjects_list.php? q=\%28PI contains Prof.\%20Dr.\%20med.\%20Markus\%20Arnold\% 29\&orderby=dBASECID [Accessed 29 Oct 2019].

26 Thienpont E, Vanden Berghe A, Schwab PE, et al. Joint awareness in osteoarthritis of the hip and knee evaluated with the 'Forgotten Joint' Score before and after joint replacement. Knee Surg Sports Traumatol Arthrosc 2016;24:3346-51.

27 Noonan VK, Lyddiatt A, Ware P, et al. Montreal Accord on PatientReported Outcomes (PROs) use series - Paper 3: patient-reported outcomes can facilitate shared decision-making and guide selfmanagement. J Clin Epidemiol 2017;89:125-35.

28 Lewis CL, Pignone MP. Promoting informed decision-making in a primary care practice by implementing decision AIDS. $N C$ Med $J$ 2009;70:136-9. 